\title{
Outcome measures and prognosis of WRMSD
}

\author{
Mr. Jerrish A Jose ${ }^{\text {a }}$ \\ Consultant in Physiotherapy and Ergonomics, RECOUP Neuromusculoskeletal Rehabilitation Centre, Bangalore, \\ INDIA
}

\begin{abstract}
Work Related Musculoskeletal Disorders (WRMSDs) are widespread in many countries, with substantial costs and impact on quality of life. WRMSDs create a significant financial burden to both employer and employee that includes the cost of treatment and lost work time. There is a growing demand to implement early identification and effective prevention strategies, aimed at early intervention in employees prior to possible absenteeism due to foreseen sickness or disability. Outcome measure of the WRMSD gives an understanding about the severity and the intensity of the problems. Before starting of the treatment it is very important to understand the gravity and seriousness of the WRMSD. Once we understand the gravity of the WRMSD the level of the treatment can be decided and optimum prescription can be made for that particular WRMSD. Objectives: This will discuss various outcome measures through which prognosis of the WRMSD can be determined and final prescription of treatment regiment can be formulated.
\end{abstract}

Keywords: NDI, DASH, Algometry, CROM

\section{Neck disability index}

The Neck Disability Index is an instrument to assess neck pain complaints. It was developed in 1989 by Howard Vernon. The Index was developed as a modification of the Oswestry Low Back Pain Disability Index. It has high reliability and validity score which was originally published by the authors and then verified by various researchers in their studies. NDI consists of one factor - "physical disability" - although NDI scores correlate well with SF-36 mental component scores as well. This has ten sections explaining Pain Intensity, Personal Care, Lifting, Reading, Headache, Concentration, Work, Driving, Sleeping and Recreation. Each of the 10 items is scored from $0-5$. The maximum score is therefore 50 . The obtained score can be multiplied by 2 to produce a percentage score. Occasionally, a respondent will not complete one question or another. The average of all other items is then added to the completed items. The NDI has become a standard instrument for measuring self-rated disability due to neck pain and is used by clinicians and researchers. It is recommended that the NDI be used at baseline and for every 2 weeks thereafter within the treatment program to measure progress. As noted above, at least a 5-point change is required to be clinically meaningful.

\section{Algometry}

Algometry is the measurement of pain by means of an algometer, an instrument for determining sensitivity to pain produced by pressure. Pressure Algometry have been marketed for diagnostic purposes in clinical practice since neuromuscular conditions are often associated with mechanical hyperalgesia. It is having a high reliability and construct validity ${ }^{2}$. Pressure algometer is basically a pressure threshold meter. The pressure threshold meter (PTM) is a force gauge with a rubber disc of 1 $\mathrm{cm}^{2}$ surface. The instrument has been proven to be useful in clinical practice for quantification of deep muscle tenderness ${ }^{1}$.

\section{Cervical range of motion}

Strength and range of motion may reduce in many patients due to their work exposure or some other acute injury. Measuring the ROM before intervention

Corresponding author, Email ID: jerrish@,recoup.in

${ }^{1}$ Address: \#312, 80 Feet Road, 10th Block, Further Extension of Anjanapura Layout, Bangalore-560062 
or treatment gives the researcher the quantitative improvement so that training programme can be more effective. An Instrumented Goniometer is used to measure the ROM.

\section{Hand held dynamometry}

To measure the grip strength and pinch strength the use of dynamometer is well established. Measure of grip strength gives a preliminary assessment of the muscle power of the patient's hand and fingers. After strength and endurance training it gives the result of quantitative improvement of these parameters.

\section{SF36}

The SF-36 $6^{\circledR}$ Health Survey is a generic outcome measure designed to examine a person's perceived health status. It's a Self-report Questionnaire. It takes 5 - 10 minute to complete. The SF-36 Health Survey includes one multi-item scale measuring each of the following eight health concepts: physical functioning, role limitations because of physical health problems, bodily pain, and social functioning; general mental health (psychological distress and psychological wellbeing), role limitations because of emotional problems, vitality (energy/fatigue) and general health perceptions.

\section{Reference}

[1] A.A. Fischer, Pressure algometry over normal muscles. Standard values, validity and reproducibility of pressure threshold, Pain, 1987, 30, 115 - 126.
The SF-36 also includes a single-item measure of health transition or change. ${ }^{2}$ The SF-36 can also be divided into two aggregate summary measures the Physical Component Summary (PCS) and the Mental Component Summary (MCS). (In the standard version of the SF-36 all scale questions refer to a 4 week time period.). The SF-36 Health Survey items and scales were constructed using the Likert method of summated ratings. Answers to each question are scored (some items need to be recoded). These scores are then summed to produce raw scale scores for each health concept which are then transformed to a $0-$ 100 scale. Scoring algorithms can then be applied to produce the PCS and MCS scores. (These two summary scores have the major advantage of being norm based. They also have reduced floor and ceiling effects.)

\section{Disability of arm, shoulder and hand questionnaire}

The DASH Outcome Measure has been increasing in popularity since its release in 1996. This questionnaire asks about the symptoms as well as the ability to perform certain activities. This is a 30 -item; self-report questionnaire designed to assess physical function and symptoms in people with any of several musculoskeletal disorders of the upper limb. The questionnaire is available in multiple languages.

[2] M. Kinser, Ann; Sands, A. William Stone, H. Michael, Reliability and Validity of a Pressure Algometer Journal of Strength \& Conditioning Research, 2009, 23 $312-314$. 